\title{
Safety and efficacy of early corticosteroid withdrawal in liver transplant recipients: new-onset diabetes after liver transplantation randomized clinical trial
}

\author{
Jong Man Kim, Jae-Won Joh¹, Kwang-Woong Lee ${ }^{2}$, Dong Lak Choi ${ }^{3}$, Hee-Jung Wang ${ }^{4}$ \\ ${ }^{1}$ Department of Surgery, Samsung Medical Center, Seoul, Korea \\ ${ }^{2}$ Department of Surgery, Seoul National University College of Medicine, Seoul, Korea \\ ${ }^{3}$ Department of Surgery, Catholic University of Daegu School of Medicine, Daegu, Korea \\ ${ }^{4}$ Department of Liver Transplantation and Hepatobiliary Surgery, Ajou University School of Medicine, Suwon, Korea
}

Background: Standard practice for immunosuppressive therapy after liver transplantation (LT) is triple therapy, tacrolimus (TAC), mycophenolate mofetil, and corticosteroid triple therapy. Long-term steroid intake significantly increases cardiovascular risk factors with negative effects on patient outcome, especially post-transplantation diabetes associated with morbidity and mortality. In this trial, we examined the efficacy and safety parameters of early steroid withdrawal during the first year after LT.

Methods: In this open-label, multicenter, randomized controlled trial, we randomly assigned LT recipients in a 1:1 ratio to receive either early corticosteroid withdrawal at 2 weeks (group 1) or corticosteroid withdrawal at 3 months (group 2) after LT. The study was performed at four centers across Korea. Only participants between 20 and 70 years of age who were scheduled to receive a single-organ liver transplant from either a living donor or a deceased donor were considered for enrollment. The primary endpoint was the incidence of new-onset diabetes after liver transplantation (NODAT) at 12 months. All analyses were done by intention-to-treat. This trial is registered with ClinicalTrials.gov, number NCT02095418.

Results: Between November 2012 to August 2020, 115 patients were randomly assigned to group $1(n=60)$ or group $2(n=55)$. The incidence of NODAT in group 1 (32.4\%) was increased compared to group $2(10.0 \%)$ in the per-protocol set. Additionally, biopsy-proven acute rejection (BPAR), graft failure, and death were not developed; however, median TAC trough level/dose/weight in group 1 were generally higher than in group 2 . Safety parameters such as infection or the incidence of hepatocellular carcinoma recurrence did not differ between the two groups.

Conclusions: Early steroid withdrawal at 2 weeks after LT shows higher NODAT development compared with steroid withdrawal at post-transplant 3 months because of generally high TAC exposure. However, early steroid withdrawal can be achieved without loss of efficacy including BPAR, graft loss, and death.

Corresponding author: Jae-Won Joh

E-mail: jw.joh@samsung.com

(C) The Korean Society for Transplantation

This is an Open Access article distributed under the terms of the Creative Commons Attribution Non-Commercial License (http://creativecommons.org/licenses/by-nc/4.0/) which permits unrestricted non-commercial use, distribution, and reproduction in any medium, provided the original work is properly cited. 